\title{
Antiplasmodial properties of kaempferol-3- $O$-rhamnoside isolated from the leaves of Schima wallichii against chloroquine-resistant Plasmodium falciparum
}

\author{
MELISA I. BARLIANA ${ }^{1}$, EKA W. SURADJI ${ }^{2,3}$, RIZKY ABDULAH ${ }^{1,3}$, AJENG DIANTINI ${ }^{1}$, \\ TOSHIMITSU HATABU ${ }^{4}$, JUNKO NAKAJIMA-SHIMADA ${ }^{4}$, ANAS SUBARNAS $^{1}$ and HIROSHI KOYAMA ${ }^{3}$ \\ ${ }^{1}$ Department of Pharmacology and Clinical Pharmacy, Faculty of Pharmacy, Universitas Padjadjaran, Jatinangor, \\ West Java 45363; ${ }^{2}$ Department of Health, Teluk Bintuni Regency, West Papua 98364, Indonesia; \\ ${ }^{3}$ Department of Public Health, Gunma University Graduate School of Medicine; \\ ${ }^{4}$ Gunma University Graduate School of Health Sciences, Maebashi, Gunma 371-8511, Japan
}

Received February 3, 2014; Accepted April 15, 2014

DOI: $10.3892 /$ br.2014.271

\begin{abstract}
Previous intervention studies have shown that the most effective agents used in the treatment of malaria were isolated from natural sources. Plants consumed by non-human primates serve as potential drug sources for human disease management due to the similarities in anatomy, physiology and disease characteristics. The present study investigated the antiplasmodial properties of the primate-consumed plant, Schima wallichii (S. wallichii) Korth. (family Theaceae), which has already been reported to have several biological activities. The ethanol extract of $S$. wallichii was fractionated based on polarity using $n$-hexane, ethyl acetate and water. The antiplasmodial activity was tested in vitro against chloroquine-resistant Plasmodium falciparum (P.falciparum) at $100 \mu \mathrm{g} / \mathrm{ml}$ for $72 \mathrm{~h}$. The major compound of the most active ethyl acetate fraction was subsequently isolated using column chromatography and identified by nuclear magnetic resonance. The characterized compound was also tested against chloroquine-resistant $P$. falciparum in culture to evaluate its antiplasmodial activity. The ethanol extract of $S$. wallichii at $100 \mu \mathrm{g} / \mathrm{ml}$ exhibited a significant parasite shrinkage after $24 \mathrm{~h}$ of treatment. The ethyl acetate fraction at $100 \mu \mathrm{g} / \mathrm{ml}$ was the most active fraction against chloroquine-resistant $P$. falciparum. Based on the structural characterization, the major compound isolated from the ethyl acetate fraction was kaempferol-3-O-rhamnoside, which showed promising antiplasmodial activity against chloroquine-resistant $P$. falciparum with an $\mathrm{IC}_{50}$ of $106 \mu \mathrm{M}$
\end{abstract}

Correspondence to: Dr Rizky Abdulah, Department of Pharmacology and Clinical Pharmacy, Faculty of Pharmacy, Universitas Padjadjaran, J1. Raya Bandung Sumedang Km 21, Jatinangor, West Java 45363, Indonesia

E-mail: abdulahrizky@gmail.com

Key words: malaria, primates, medicinal plant, natural product, antiplasmodial after $24 \mathrm{~h}$ of treatment. The present study has provided a basis for the further investigation of kaempferol-3- $O$-rhamnoside as an active compound for potential antimalarial therapeutics.

\section{Introduction}

Malaria is an infectious disease endemic throughout tropical countries. Malaria is also prevalent in subtropical areas, where the disease is contagious affecting both indigenous population and travelers (1). Malaria is caused by Plasmodium parasites that are transmitted through the bite of Anopheles mosquitoes and have a life cycle in mosquito and human hosts (1). Of all parasite types, Plasmodium falciparum ( $P$. falciparum) is the most dangerous Plasmodium, causing human malaria with a mortality of 1-2 million people annually. According to surveys conducted between 1900 and 2008 in 2,366 locations in Indonesia, four species of Plasmodium may infect humans, $P$. falciparum, $P$. vivax, $P$. malariae and $P$. ovale. $P$. falciparum is the most common parasite that is contagious in Indonesia, with prevalence rates of 33\% in Papua, 29\% in Lesser Sundas and $21 \%$ in Sumatra (2). Findings of studies performed in other parts of Indonesia, including the Thousand Island district (3), Nias Island (4), Sumba Island (5) and Aceh (6), have shown that $P$.falciparum was the most frequent parasite that caused malaria.

Eradication of malaria remains challenging due to drug resistance of Plasmodium. Hyde (1) reported that the first synthetic antimalarial drug, found in the 1930s, was chloroquine, which was highly effective, safe and cost-effective. Since 1957, however, resistance to administration of chloroquine was observed in Thailand and by 1988 this resistance had spread to sub-Saharan Africa and other areas of the world. Several factors affect antimalarial resistance, including the overuse of drugs for prophylaxis, incomplete therapeutic treatments of active infections, genetic and metabolic adaptive abilities of the parasites and a massive parasite proliferation (1). The incidence of malaria infections, which is 250 million cases and 80,000 mortalities annually, has revealed the emerging requirement for identifying new classes of medicine (7). 
Medicinal plants have been used as traditional medicines for hundreds of years. The first antimalarial agents, including quinine as well as the next generation of antimalarial agents, such as lapachol and artemisinin, have been isolated from plants $(7,8)$. Traditional medicines have a high potential as novel drug candidates, can provide valuable clues to find novel drugs and may shift the drug discovery paradigm from 'finding new-entity drugs' to 'combining existing agents' $(9,10)$. Certain approaches in finding novel drugs use plants that are consumed by particular groups (11). Primates have a close similarity with human physiology and also have similar characteristics of disease as humans. Humans use drugs to cure these diseases, whereas primates can only rely on the foods they eat to protect themselves against these diseases.

Due to the high degree of physiological similarity between primates and humans, primate disease often exhibits similarities to human disease, including cancer and malaria. Notably, certain human diseases are known to have originated from primates (11). As the survival rates of primates is mainly dependent on daily food intake, the food consumed is considered to be a promising source of products applicable for the management of human disease. In a previous study, 19 primate-consumed plants were collected and their anti-tumor promoting activity was confirmed in vitro (11), with two species, Kadsura scandens (Blume) Blume (family Schisandraceae) and Schima wallichii (S. wallichii) (family Theaceae), showing antimutagenic activities in further investigations (12). The potential of $S$. wallichii to inhibit MCF-7 breast cancer cell proliferation has also been reported (13). Findings of an ethnobotanical study in Mizoram showed that S. wallichii is used for snake and insect bites (14). S. wallichii is a tree with a height of 5-30 m and is usually found in tropical countries, including Indonesia, the Philippines, Nepal, Sikkim, Assam, Myanmar, South China and the Malay Peninsula (15). In the present study, the antiplasmodial properties of the $S$. wallichii leaves were investigated and its active compound was identified.

\section{Materials and methods}

Plant collection. S. wallichii leaves were collected from the Pangandaran Beach conservation area in the West Java province of Indonesia. The leaf of S. wallichii was identified in the School of Biological Science and Technology, Bandung Institute of Technology, Bandung, Indonesia.

Extraction, fractionation and isolation. The $S$. wallichii leaves were dried and extracted with $70 \%$ ethanol at room temperature three times for $24 \mathrm{~h}$ each. A concentrated extract was obtained in vacuo at $50^{\circ} \mathrm{C}$. The ethanol extract $(86.94 \mathrm{~g})$ was partitioned into $n$-hexane $(3.03 \mathrm{~g})$, ethyl acetate $(3.27 \mathrm{~g})$ and aqueous phases (7.19 g), respectively. Column chromatography on a Wakogel C-200 (Wako Pure Chemical Industries, Ltd., Chuo-ku, Osaka, Japan) column was performed on the most active ethyl acetate fraction, using a mixture of $n$-hexane, ethyl acetate and methanol with increasing polarity. The major compound observed was purified using silica G-60 with sulfuric acid-ethanol (1:9) and was found to be the most active fraction of S. wallichii, which was characterized and analyzed as described previously (13). The isolate was, however, identi- fied by spectroscopic methods [ultraviolet, infrared, nuclear magnetic resonance (NMR)] and liquid chromatography mass spectrometry (16).

P. falciparum parasite culture. The chloroquine-resistant $P$. falciparum strain, K-1, was cultured asynchronously as described in a previous study (17). The culture was grown in RPMI-1640 medium (Sigma-Aldrich, St. Louis, MO, USA) containing $10 \%$ type B or O human serum (serum type showed no significant effect on parasite growth), $25 \mathrm{mM}$ 4-(2-hydroxyethyl)-1-piperazineethanesulfonic acid (HEPES) (Wako Pure Chemical Industries, Ltd.), $25 \mu \mathrm{g} / \mathrm{ml}$ gentamycin (Sigma-Aldrich), $25 \mathrm{mM}$ sodium bicarbonate (Wako Pure Chemical Industries, Ltd.) and human type O red blood cells (RBCs) to generate the final 5\% hematocrit mixture. The parasite was cultured in a humidified incubator in $5 \% \mathrm{CO}_{2}$ and $5 \% \mathrm{O}_{2}$ at $37^{\circ} \mathrm{C}$.

Growth inhibitory assay. The growth inhibitory effect of the extract, fractions or isolate of $S$. wallichii against $P$. falciparum was determined by culturing the parasite with an initial parasitemia of $0.1 \%$ in $5 \%$ hematocrit. The culture medium containing the extract, fractions or isolate of $S$. wallichii was changed every $24 \mathrm{~h}$ and the number of RBCs containing the parasite (pRBCs) was counted. Thin-smeared slides of Giemsa-stained RBCs were prepared, pRBCs were counted, and the number of pRBCs in 3,000 RBCs was determined under a light microscope at a magnification of $\mathrm{x} 1,000$. The experiment was terminated at $72 \mathrm{~h}$. Each concentration was created in triplicate and the experiment was performed in triplicate. The parasite morphology was observed prior to counting the pRBCs in the same culture plate using the same method.

Determination of the $I C_{50}$ concentration against $P$. falciparum. The $\mathrm{IC}_{50}$ determination of kaempferol-3-O-rhamnoside against $P$. falciparum was performed using a similar method to that of the 'growth inhibitory assay', as described in a previous report (18). The culture medium containing kaempferol-3-O-rhamnoside with concentrations of 115,230 and $460 \mu \mathrm{M}$ was incubated for $24 \mathrm{~h}$ and the number of pRBCs was counted. Thin-smeared slides of Giemsa-stained RBCs were prepared, the $\mathrm{pRBCs}$ were counted, and the number of pRBCs in 3,000 RBCs was determined under a light microscope at a magnification of $x 1,000$. The $\mathrm{IC}_{50}$ values that represent the concentrations required to inhibit $50 \%$ of plasmodium growth were calculated from a calibration curve by linear regression.

Statistical analysis. Statistically significant differences were determined by the Student's t-test. $\mathrm{P}<0.05$ was considered to indicate a statistically significant difference.

\section{Results}

Growth of P. falciparum is inhibited by the ethyl acetate fraction of S. wallichii. The $S$. wallichii extract $(100 \mu \mathrm{g} / \mathrm{ml})$ was shown to inhibit $P$. falciparum growth after a $24-\mathrm{h}$ treatment. P. falciparum morphological changes were detected following treatment with the extract (Fig. 1). The extract was partitioned into $n$-hexane, ethyl acetate and water. The extract and each 
A

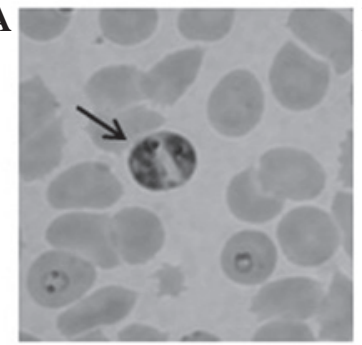

B

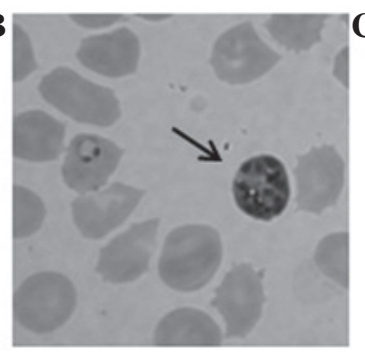

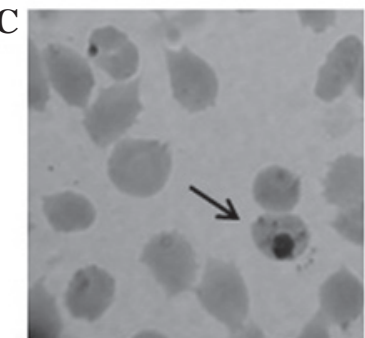

Figure 1. Plasmodium falciparum morphology after 24 h of incubation with the Schima wallichii (S. wallichii) Korth. extract. (A) No change was observed without treatment or control (arrow), while changes were detected following incubation with (B) 10 or (C) $100 \mu \mathrm{g} / \mathrm{ml}$ extract as indicated by the arrows. The treatment with $100 \mu \mathrm{g} / \mathrm{ml} \mathrm{S.} \mathrm{wallichii} \mathrm{Korth.} \mathrm{extract} \mathrm{caused} \mathrm{shrinkage} \mathrm{and} \mathrm{pyknotic} \mathrm{bodies} \mathrm{in} \mathrm{the} \mathrm{parasite} \mathrm{morphology.}$

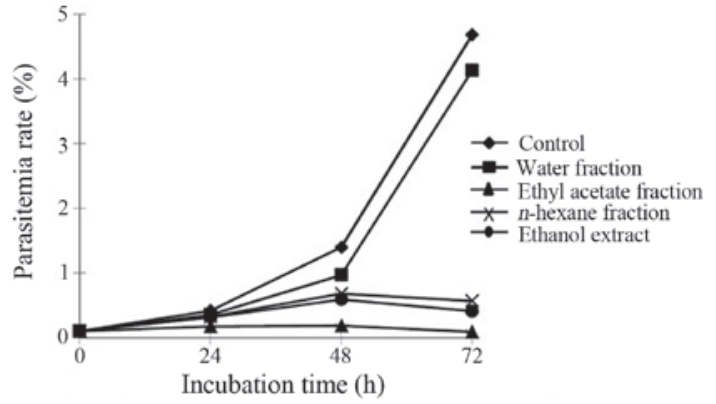

Figure 2. Growth inhibitory effect against Plasmodium falciparum (P.falciparum) following treatment with the Schima wallichii ( $S$. wallichii) Korth. extract and fractions for $24-72 \mathrm{~h}(100 \mu \mathrm{g} / \mathrm{ml}$ each). The ethyl acetate fraction of $S$. wallichii Korth. showed the strongest inhibitory effect on the P. falciparum growth rate. Data are the mean of three independent experiments.<smiles>CC(O)C1C(O)C(O)C(Oc2c(-c3ccc(O)cc3)oc3cc(O)cc(O)c3c2=O)C1O</smiles>

Figure 3. Structure of kaempferol-3- $O$-rhamnoside (or 3,4',5,7-tetrahydroxyflavone-3-O-rhamnoside) isolated from Schima wallichii Korth. leaves.

fraction at a concentration of $100 \mu \mathrm{g} / \mathrm{ml}$ were then used to treat the parasites cultured in RBCs for $72 \mathrm{~h}$. The extract, $n$-hexane and ethyl acetate fractions significantly inhibited parasite growth in the RBC culture, which was observed by the decreasing parasitemia level of 3,000 RBCs compared with that in the untreated parasite culture (Fig. 2). These results indicated that the ethyl acetate fraction was the most active fraction that inhibited parasite growth.

Kaempferol-3-O-rhamnoside is the major compound of the ethyl acetate fraction of the $S$. wallichii extract. The strongest parasite growth inhibition effect was observed following the treatment with the ethyl acetate fraction of S. wallichii. Based on a previous study (13), it was found that the major compound of the ethyl acetate fraction of the S. wallichii extract was

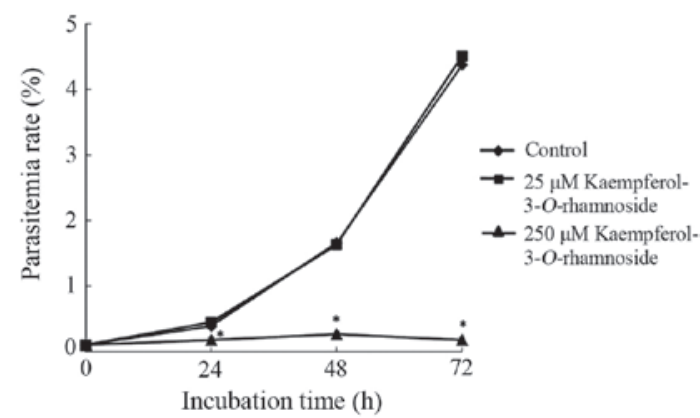

Figure 4. Antiplasmodial activity of kaempferol-3- $O$-rhamnoside against Plasmodium falciparum. Treatment with $250 \mu \mathrm{M}$ kaempferol-3-O-rhamnoside showed strong antiplasmodial activity. Data are the mean of three independent experiments. ${ }^{*} \mathrm{P}<0.05$ compared to control.

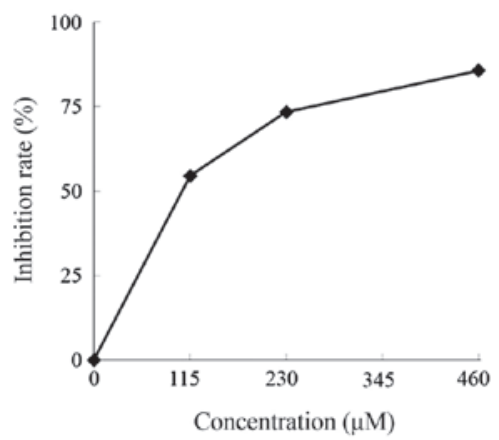

Figure 5. Various concentrations of kaempferol-3-O-rhamnoside determining the $\mathrm{IC}_{50}$ value against Plasmodium falciparum after incubation for $24 \mathrm{~h}$. The $\mathrm{IC}_{50}$ value of kaempferol-3-O-rhamnoside was $106 \mu \mathrm{M}$. Data are the mean of three independent experiments.

kaempferol-3-O-rhamnoside. The compound was purified, isolated and identified as kaempferol-3-O-rhamnoside $\left(\mathrm{C}_{21} \mathrm{H}_{20} \mathrm{O}_{10}\right)$ with a molecular weight of 432 (Fig. 3).

Kaempferol-3-O-rhamnoside inhibits $P$. falciparum culture growth. The antiplasmodial effect of kaempferol-3-O-rhamnoside was observed 24-72 $\mathrm{h}$ following the treatment with kaempferol-3-O-rhamnoside in $P$. falciparum culture (Fig. 4). Parasite growth was significantly inhibited by $250 \mu \mathrm{M}$ kaempferol-3-O-rhamnoside to $54.3 \%$ at $24 \mathrm{~h}$ of treatment, $83.9 \%$ at $48 \mathrm{~h}$ and $96 \%$ at $72 \mathrm{~h}$ compared with the untreated control. The results shown in Fig. 4 also show that the antiplasmodial properties of kaempferol-3-O-rhamnoside were dose-dependent. 
Incubation with $25 \mu \mathrm{M}$ kaempferol-3-O-rhamnoside did not show significant parasite growth inhibitory activity (almost similar to the negative control).

Subsequent analysis of the antiplasmodial activities against P. falciparum culture using various concentrations $(0,115$, 230,345 and $460 \mu \mathrm{M}$ ) of kaempferol-3- $O$-rhamnoside for $24 \mathrm{~h}$ showed that the $\mathrm{IC}_{50}$ value of kaempferol-3- $O$-rhamnoside was $106 \mu \mathrm{M}$ (Fig. 5). At this concentration, kaempferol-3- $O$-rhamnoside actively inhibited the growth rate of P.falciparum and selectively affected the parasite, but not the human RBCs as the host cells.

\section{Discussion}

In the present study, treatment with $100 \mu \mathrm{g} / \mathrm{ml} \mathrm{S}$. wallichii extract caused shrinkage and pyknotic bodies in the parasite morphology, but not in the human RBCs as the host cells in $P$. falciparum culture. Pyknosis is an irreversible condition involving chromatin condensation in the nucleus. This condition shows ongoing necrosis or apoptosis inside the cells, which is followed by nucleus fragmentation (19). This finding showed that $S$. wallichii Korth. extract has selective antimalarial activity.

The results also demonstrate that the ethanol extract of $S$. wallichii inhibited parasite growth. Results of the present study also show that the ethyl acetate fraction of $S$. wallichii had the strongest antiplasmodial activity in a time-dependent manner. This result suggests that this fraction has the potential to be explored for its active antiplasmodial compounds.

The characterized compound in the ethyl acetate fraction was determined to be kaempferol-3- $O$-rhamnoside following purification by column chromatography and identification by NMR. In addition, kaempferol-3- $O$-rhamnoside that was isolated from $S$. wallichii has also been reported to have anti-cancer activity by inducing the apoptotic mechanism through the caspase-cascade pathway in MCF-7 breast cancer cells (13). The results of the present study show that at a concentration of $250 \mu \mathrm{M}$, kaempferol-3-O-rhamnoside inhibited parasite growth in a time-dependent manner. In subsequent investigations, the $\mathrm{IC}_{50}$ was also confirmed to be $106 \mu \mathrm{M}$ in vitro against chloroquine-resistant $P$. falciparum after the 24-h treatment.

Oxidative stress through the generation of reactive oxygen species (ROS) plays an important role in the pathogenesis of malarial infection. During rapid growth and multiplying, plasmodium produces toxic redox active by-products that cause host haemoglobin degradation (20-22). Additionally, ROS are also produced by recruited and activated monocytes and neutrophils during infections that attack infected and uninfected erythrocytes, which increases the ROS level (22-24). Plasmodium cause damage in the membrane cells of infected and uninfected erythrocytes by inducing lipid peroxidation, leading to an aging-like process, eventually resulting in anemia (24). As a polyphenol, kaempferol-3-O-rhamnoside is able to inhibit lipid peroxidation and cyclooxygenase (COX) enzymes (COX-1 and COX-2) (25). Thus, it can be hypothesized that these antioxidant properties may be responsible for the antiplasmodial acitivity of kaempferol-3-O-rhamnoside.

The present study has shown the antiplasmodial activity of S. wallichii, which was previously reported for its anticancer properties (13). This is similar to another study, which focused on Cryptolepis sanguinolenta (Lindl.) Schltr (Periplocaceae), a traditional antimalarial in West Africa, for its anticancer activity by its cytotoxic effect in mammalian cells (26).

As the study is in a preliminary stage, follow-up studies on the antiplasmodial mechanisms in various plasmodium life stages in vitro an in vivo are currently being conducted in our laboratory. However, these findings provide a basis for subsequent investigations of kaempferol-3- $O$-rhamnoside as a candidate compound for potential antimalarial in drug development.

\section{References}

1. Hyde JE: Drug-resistant malaria - an insight. FEBS J 274: 4688-4698, 2007.

2. Elyazar IR, Hay SI and Baird JK: Malaria distribution, prevalence, drug resistance and control in Indonesia. Adv Parasitol 74: 41-175, 2011.

3. Maguire JD, Tuti S, Sismadi P, et al: Endemic coastal malaria in the Thousand Islands District, near Jakarta, Indonesia. Trop Med Int Health 10: 489-496, 2005.

4. Syafruddin D, Asih PB, Wahid I, et al: Malaria prevalence in Nias District, North Sumatra Province, Indonesia. Malar J 6: 116, 2007.

5. Asih PB, Rogers WO, Susanti AI, et al: Seasonal distribution of anti-malarial drug resistance alleles on the island of Sumba, Indonesia. Malar J 8: 222, 2009.

6. Asih PB, Rozi IE, Herdiana, et al: The baseline distribution of malaria in the initial phase of elimination in Sabang Municipality, Aceh Province, Indonesia. Malar J 11: 291, 2012.

7. Wells TN: Natural products as starting points for future anti-malarial therapies: going back to our roots? Malar J 10 (Suppl 1): S3, 2011.

8. Ginsburg H and Deharo E: A call for using natural compounds in the development of new antimalarial treatments - an introduction. Malar J 10 (Suppl 1): S1, 2011.

9. Kong DX, Li XJ and Zhang HY: Where is the hope for drug discovery? Let history tell the future. Drug Discov Today 14: 115-119, 2009.

10. Wagner H and Ulrich-Merzenich G: Synergy research: approaching a new generation of phytopharmaceuticals. Phytomedicine 16: 97-110, 2009.

11. Koshimizu K, Murakami A, Hayashi H, Ohigashi H, Subarnas A, Gurmaya KJ and Ali A: Biological activities of edible and medicinal plants from Indonesia and Malaysia. In: Proceedings of The Tokyo International Forum on Conservation and Sustainable Use of Tropical Bioresources. Japan Bioindustry Association, Tokyo, pp203-208, 1998.

12. Subarnas A, Hadiansyah C, Gurmaya KJ and Muhtadi A: Characterization of antimutagenic compound from primatesconsumed plant Schima wallichii. Biotika 2: 7-13, 2003.

13. Diantini A, Subarnas A, Lestari K, et al: Kaempferol-3-Orhamnoside isolated from the leaves of Schima wallichii Korth. inhibits MCF-7 breast cancer cell proliferation through activation of the caspase cascade pathway. Oncol Lett 3: 1069-1072, 2012.

14. Lalfakzuala $R$, Lalramnghinglova $H$ and Kayang $H$ : Ethnobotanical usages of plants in western Mizoram. Indian J Tradit Knowl 6: 486-493, 2007.

15. Keng H: Flora malesianae precursores - LVIII, part four. The genus Schima (Theaceae) in Malesia. Gard Bull Singapore 46: 77-88, 1994.

16. Silverstein RM, Webster FX and Kiemle DJ (eds): Spectrometric Identification of Organic Compounds. 7th edition. John Wiley \& Sons, New Jersey, NJ, pp72-229, 2005.

17. Taguchi N, Hatabu T, Yamaguchi H, Suzuki M, Sato K and Kano S: Plasmodium falciparum: selenium-induced cytotoxicity to P.falciparum. Exp Parasitol 106: 50-55, 2004.

18. Suradji EW, Hatabu T, Kobayashi K, et al: Selenium-induced apoptosis-like cell death in Plasmodium falciparum. Parasitology 19: 1-11, 2011.

19. Baehrecke EH: How death shapes life during development. Nat Rev Mol Cell Biol 3: 779-787, 2002.

20. Ginsburg H and Atamna H: The redox status of malaria-infected erythrocytes: an overview with an emphasis on unresolved problems. Parasite 1: 5-13, 1994. 
21. Becker K, Tilley L, Vennerstrom JL, Roberts D, Rogerson S and Ginsburg H: Oxidative stress in malaria parasite-infected erythrocytes: host-parasite interactions. Int J Parasitol 34: 163-189, 2004.

22. Ibrahim MA, Zuwahu MM, Isah MB, Jatau ID, Aliyu AB and Umar IA: Effects of vitamin E administration on Plasmodium berghei induced pathological changes and oxidative stress in mice. Trop Biomed 29: 98-106, 2012.

23. Postma NS, Mommers EC, Eling WM and Zuidema J: Oxidative stress in malaria: implications for prevention and therapy. Pharm World Sci 18: 121-129, 1996.
24. Omodeo-Salè F, Motti A, Basilico N, Parapini S, Olliaro P and Taramelli D: Accelerated senescence of human erythrocytes cultured with Plasmodium falciparum. Blood 102: 705-711, 2003.

25. Vareed SK, Schutzki RE and Nair MG: Lipid peroxidation, cyclooxygenase enzyme and tumor cell proliferation inhibitory compounds in Cornus kousa fruits. Phytomedicine 14: 706-709, 2007.

26. Ansah C and Mensah KB: A review of the anticancer potential of the antimalarial herbal Cryptolepis sanguinolenta and its major alkaloid cryptolepine. Ghana Med J 47: 137-147, 2013. 